Guowei Kim, Hon Lyn Tan, Elya Chen, Siok Chin Teo, Clarisse Jia Min Jang, Jingshan Ho, Yvonne Ang, Natalie Yan Li Ngoi, Cheng Ean Chee, Bettina Lieske, Asim Shabbir, Ling-Zhi Wang, Jimmy Bok Yan So* and Wei Peng Yong

\title{
Study protocol: phase 1 dose escalating study of Pressurized Intra-Peritoneal Aerosol Chemotherapy (PIPAC) with oxaliplatin in peritoneal metastasis
}

https://doi.org/10.1515/pap-2018-0118

Received May 25, 2018; accepted August 02, 2018;

previously published online August 29, 2018

\section{Abstract}

Background: Pressurized Intra-Peritoneal Aerosol Chemotherapy (PIPAC) is a novel laparoscopic intraperitoneal chemotherapy technique, with advantages such as homogeneous distribution of aerosol and deeper tissue penetration. Thus far, PIPAC oxaliplatin has been administered at an arbitrary dose of $92 \mathrm{mg} / \mathrm{m}^{2}$.

Aim: We aim to determine the dose-related safety profile and tolerability of PIPAC oxaliplatin using an evidencebased approach. The secondary aim is to evaluate clinicpathologic response and the pharmacokinetic profile.

\footnotetext{
*Corresponding author: Jimmy Bok Yan So, Department of Surgery, National University of Singapore, Yong Loo Lin School of Medicine, Singapore, Singapore, E-mail: jimmy_so@nuhs.edu.sg

Guowei Kim, University Surgical Cluster, National University Health System, Singapore, Singapore, E-mail: guo_wei_kim@nuhs.edu.sg http://orcid.org/0000-0002-4705-6808

Hon Lyn Tan, Department of Hematology-Oncology, National

University Cancer Institute, Singapore, Singapore,

E-mail: hon_lyn_tan@nuhs.edu.sg

Elya Chen: E-mail: surel@nus.edu.sg, Siok Chin Teo:

E-mail: surtsc@nus.edu.sg, Clarisse Jia Min Jang:

E-mail: surjjmc@nus.edu.sg, Department of Surgery, National University of Singapore, Yong Loo Lin School of Medicine, Singapore, Singapore

Jingshan Ho: E-mail: jingshan_ho@nuhs.edu.sg, Yvonne Ang: E-mail: yvonne_ang@nuhs.edu.sg, Natalie Yan Li Ngoi: E-mail: natalie_yl_ngoi@nuhs.edu.sg, Cheng Ean Chee: E-mail: cheng_ean_chee@nuhs.edu.sg, Department of HematologyOncology, National University Cancer Institute, Singapore, Singapore

Bettina Lieske: E-mail: bettina_lieske@nuhs.edu.sg, Asim Shabbir E-mail:asim_shabbir@nuhs.edu.sg, University Surgical Cluster, National University Health System, Singapore, Singapore Ling-Zhi Wang, Cancer Science Institute of Singapore, Singapore, Singapore, E-mail: csiwl@nus.edu.sg

Wei Peng Yong, Department of Hematology-Oncology, National University Cancer Institute, Singapore, Singapore,

E-mail: Wei_Peng_YONG@nuhs.edu.sg
}

Methods: This is a phase I $3+3$ dose escalation study for gastric and colorectal cancer with predominant peritoneal metastasis starting at a dose of $45 \mathrm{mg} / \mathrm{m}^{2}$. Safety is assessed according to Clavien-Dindo Classification and Common Terminology Criteria for Adverse Events (version 4.0). Clinico-pathologic response is assessed using the Peritoneal Regression Grading Score, Peritoneal Cancer Index, and Response Evaluation Criteria In Solid Tumour criteria (version 1.1). Pharmacokinetic analysis is performed using Inductively Coupled Plasma-Mass Spectrometry assay. This trial is registered on ClinicalTrials.gov (NCT03172416).

Conclusions: This phase I study can provide the scientific basis to identify the optimal dose for PIPAC with oxaliplatin such that the benefits of this novel and promising intraperitoneal chemotherapy delivery technique can be maximized.

Keywords: dose escalation, oxaliplatin, peritoneal carcinomatosis, phase I, PIPAC, study protocol

\section{Introduction}

Pressurized Intra-Peritoneal Aerosol Chemotherapy (PIPAC) is an innovative IP chemotherapy concept for treating peritoneal metastasis that enhances efficacy by taking advantage of the physical properties of gas and pressure [1]. This results in a superior distribution and depth of penetration of the drug $[1,2]$. To date, most phase II trials utilizing PIPAC involve the use of cisplatin and doxorubicin [3-5]. Oxaliplatin is an approved drug for systemic chemotherapy, with well documented use intraperitoneally via hyperthermic intraperitoneal chemotherapy (HIPEC) as well. This makes it a favourable agent for PIPAC in early phase studies. Only three prior studies have utilized oxaliplatin in PIPAC for peritoneal metastasis $[4,6,7]$. However, the dose of oxaliplatin utilized for PIPAC in these studies was arbitrarily set at $92 \mathrm{mg} / \mathrm{m}^{2}$, which is approximately $20 \%$ of the dose used in HIPEC. Furthermore, some of these studies were 
performed on patients with a recent or concurrent administration of systemic chemotherapy, which may make interpretation of the side effects and safety profile difficult to interpret.

In this study, we intend to determine the dose-related safety profile and tolerability of PIPAC with oxaliplatin by assessment of dose-limiting toxicities (DLT) and the adverse event profile via an evidence-based approach. The secondary aim is to evaluate the clinical and pathological response of PIPAC with oxaliplatin as well as to identify the pharmacokinetic profile of oxaliplatin administered via PIPAC.

\section{Methods and design}

This is a prospective, single arm phase I trial in a $3+3$ dose escalation design (Figure 1) evaluating the safety and tolerability of PIPAC using oxaliplatin in patients with peritoneal metastasis. Ethics approval was obtained from the National Healthcare Group Domain Specific Review Board (DSRB) (2016/01088). This trial is also registered on ClinicalTrials.gov. (NCT03172416).

\section{Patient selection and study population}

Eligible patients should have gastric and colorectal cancers with unresectable peritoneal metastasis. The protocol was recently expanded to include patients with unresectable peritoneal metastasis from other primary tumours. Eligibility for recruitment would be based on the inclusion and exclusion criteria (Table 1). They should have undergone or have been offered at least first-line systemic chemotherapy, as PIPAC is still investigational with no phase III studies demonstrating superiority over first-line systemic chemotherapy.

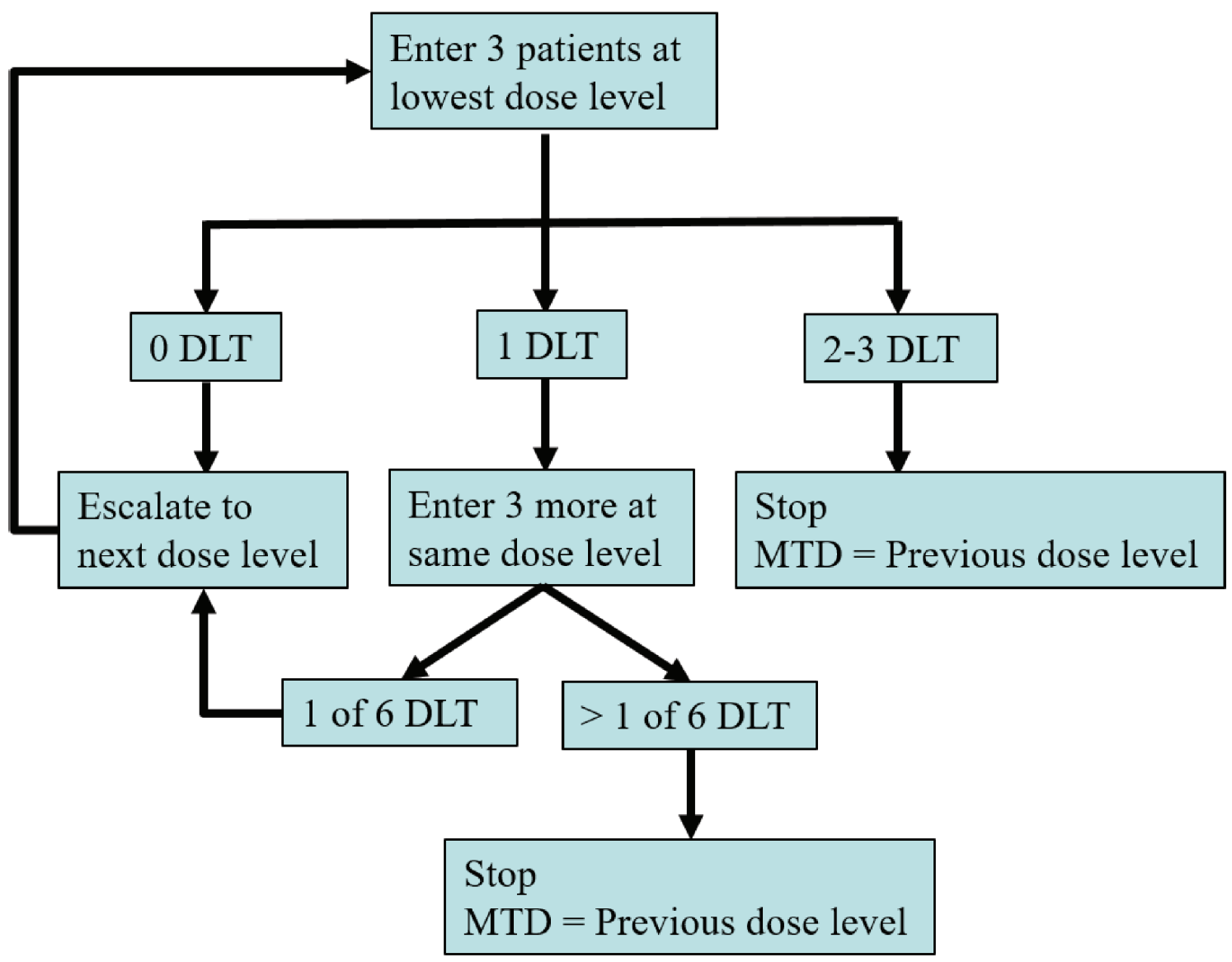

DLT $=$ Dose Limiting Toxicity

MTD = Maximum Tolerable Dose

Figure 1: The $3+3$ dose escalation study design.

DLT, dose limiting toxicity; MTD, maximum tolerable dose. 
Table 1: Inclusion and exclusion criteria.

\begin{tabular}{|c|c|}
\hline Inclusion criteria & Exclusion criteria \\
\hline $\begin{array}{l}\text { All cancer patients with unresectable peritoneal metastasis on } \\
\text { peritoneal cytology/histology }\end{array}$ & $\begin{array}{l}\text { Predominant extra-peritoneal metastases at the discretion of the study } \\
\text { team after discussion at the multidisciplinary tumour board }\end{array}$ \\
\hline $\begin{array}{l}\text { Patients who refuse, are unable to tolerate, or have completed at } \\
\text { least first-line systemic chemotherapy }\end{array}$ & $\begin{array}{l}\text { Good response to systemic chemotherapy based on RECIST guidelines } \\
\text { version } 1.1 \text {, with complete or partial response to systemic } \\
\text { chemotherapy }\end{array}$ \\
\hline $\begin{array}{l}\text { Patients who have completed chemotherapy/targeted } \\
\text { therapy }>21 \text { days or at least } 5 \text { half-lives (or whichever is longer) } \\
\text { prior to PIPAC }\end{array}$ & Known allergy to oxaliplatin \\
\hline Age $>21$ years & $\begin{array}{l}\text { Previous malignancy unrelated to current peritoneal metastasis } \\
\text { diagnosed in the last } 5 \text { years }\end{array}$ \\
\hline Eastern Cooperative Oncology Group performance status $0-3$ & $\begin{array}{l}\text { Significant disease or conditions which, in the investigator's opinion, } \\
\text { would exclude patient from the study }\end{array}$ \\
\hline $\begin{array}{l}\text { Adequate bone marrow function (neutrophil count }>1,500 / \mathrm{mm} 3 \text {, } \\
\text { haemoglobin }>8.0 \mathrm{~g} / \mathrm{dl} \text { and platelet count }>100,000 / \mathrm{mm}^{3} \text { ) }\end{array}$ & $\begin{array}{l}\text { Uncontrolled intercurrent illness including, but not limited to, ongoing } \\
\text { or active infection, symptomatic congestive heart failure, unstable } \\
\text { angina pectoris, cardiac arrhythmia, or psychiatric illness/social } \\
\text { situations that would limit compliance with study requirements }\end{array}$ \\
\hline $\begin{array}{l}\text { Adequate liver function (bilirubin, AST/ALT within upper limit of } \\
\text { normal) }\end{array}$ & $\begin{array}{l}\text { Patients with reproductive potential who refuse to use an adequate } \\
\text { means of contraception (including male patients) }\end{array}$ \\
\hline $\begin{array}{l}\text { Adequate renal function (serum creatinine within the upper limit } \\
\text { of normal) }\end{array}$ & Pregnant or lactating female \\
\hline Expected survival $>3$ months & \\
\hline
\end{tabular}

\section{Screening and enrolment}

Potential subjects will be identified by the study team with clinicians from the study team making the first contact with subjects. The clinician will assess patient suitability and eligibility for primary registration for the study and inform the patient about this clinical trial. The study team will then obtain written informed consent by means of a dated signature from the potential subjects with the original being retained by the study team. Screening/baseline evaluations are to be conducted within 4 weeks prior to start of PIPAC. Details on the evaluations required can be found in the study schedule (Table 2).

Table 2: Study schedule.

\begin{tabular}{|c|c|c|c|c|c|c|c|c|c|c|c|c|c|}
\hline \multirow{2}{*}{$\begin{array}{l}\text { Assessment } \\
\text { Week }\end{array}$} & \multirow[t]{2}{*}{ Screening } & \multicolumn{4}{|c|}{ PIPAC 1} & \multicolumn{2}{|c|}{$F / U$ for $A E$} & \multicolumn{3}{|c|}{ PIPAC 2} & & \multicolumn{2}{|c|}{$F / U$ for $A E$} \\
\hline & & 1 & & & & 3 & 6 & 7 & & & & 9 & 12 \\
\hline Day no. & -28 to 0 & 1 & 2 & 3 & 4 & 15 & 36 & 43 & 44 & 45 & 46 & 57 & 78 \\
\hline Informed consent & $\square$ & & & & & & & & & & & & \\
\hline Medical history and demographic & $\square$ & & & & & & & & & & & & \\
\hline Clinico-surgical findings & & $\square$ & & & & & & $\square$ & & & & & \\
\hline Physical examination & $\square$ & $\square$ & $\square$ & $\square$ & $\square$ & $\square$ & $\square$ & $\square$ & $\square$ & $\square$ & $\square$ & $\square$ & $\square$ \\
\hline Vital signs (height at screening only) & $\square$ & $\square$ & $\square$ & $\square$ & $\square$ & $\square$ & $\square$ & $\square$ & $\square$ & $\square$ & $\square$ & $\square$ & $\square$ \\
\hline Performance status & $\square$ & & & & & & $\square$ & & & & & & \\
\hline 12-lead ECG & $\square$ & & & & & & & & & & & & \\
\hline $\mathrm{FBC}$ & $\square$ & $\square$ & $\square$ & $\square$ & $\square$ & $\square$ & $\square$ & $\square$ & $\square$ & $\square$ & $\square$ & $\square$ & $\square$ \\
\hline Biochemistry & $\square$ & $\square$ & $\square$ & $\square$ & $\square$ & $\square$ & $\square$ & $\square$ & $\square$ & $\square$ & $\square$ & $\square$ & $\square$ \\
\hline Pharmacokinetic assessment & & $\square$ & $\square$ & & & & & $\square$ & $\square$ & & & & \\
\hline Ascites/peritoneal washing & $\square$ & $\square$ & & & & & & $\square$ & & & & & \\
\hline Serum or urine pregnancy test & $\square$ & & & & & & & & & & & & \\
\hline Clinical toxicity assessments & & $\square$ & $\square$ & $\square$ & $\square$ & $\square$ & $\square$ & $\square$ & $\square$ & $\square$ & $\square$ & $\square$ & $\square$ \\
\hline EORTC QLQ-C30 & $\square$ & & & & & & $\square$ & & & & & & $\square$ \\
\hline $\mathrm{CT}$ imaging & $\square$ & & & & & & $\square$ & & & & & & $\square$ \\
\hline
\end{tabular}




\section{PIPAC administration}

PIPAC will be administered as described by Solass et al. [5], using oxaliplatin reconstituted in $150 \mathrm{~mL}$ of Dextrose solution administered at a flow rate of $30 \mathrm{~mL} / \mathrm{min}$ with a maximal upstream pressure of 200 psi. The system was kept at a steady-state for a total of $30 \mathrm{~min}$ at an intraabdominal pressure of $12 \mathrm{mmHg}$.

Treatment will be administered on an inpatient basis. No investigational or commercial agents or therapies other than those described below may be administered with the intent to treat the patient's malignancy. Patients with a weight increase or decrease of more than $10 \%$ from that recorded at baseline within 3 days prior to study treatment on day 1 of all subsequent cycles, which is clearly not related to fluid retention, should have their body surface area (BSA) recalculated and dosage of oxaliplatin should be re-adjusted. A delay of up to 14 days for subsequent PIPAC is allowed in this study for any reason other than an adverse event, which must be reported.

A recent amendment to the protocol includes flexibility for the investigator to add Intravenous 5-Fluorouracil for the second PIPAC procedure in subjects who do not experience DLT or treatment-related serious adverse events (SAE) from the first PIPAC procedure.

After two PIPAC procedures, the study subjects may continue with further PIPAC treatments at the investigator's discretion, off-trial.

\section{Rationale for doses selected}

In one of the phase II trials which performed 48 PIPAC procedures on 17 patients exclusively with oxaliplatin[6], $23 \%$ of patients experienced Common Terminology Criteria for Adverse Events Version 4.0 (CTCAE) grade 3 adverse events with $64 \%$ experiencing abdominal pain, $23 \%$ fever, $41 \%$ nausea, and $6 \%$ diarrhoea. Pathological response was seen in $71 \%$ ( 7 complete, 4 major, and 1 partial response) of patients. As the dose of $92 \mathrm{mg} / \mathrm{m}^{2}$ has shown good pathologic response despite a large proportion of minor (CTCAE grade 1 or 2) adverse events, we aim to perform a dose-finding study starting at a lower dose of $45 \mathrm{mg} / \mathrm{m}^{2}$. In total, five cohorts are planned at the PIPAC oxaliplatin doses of $45 \mathrm{mg} / \mathrm{m}^{2}, 60 \mathrm{mg} / \mathrm{m}^{2}, 90 \mathrm{mg} / \mathrm{m}^{2}$, $120 \mathrm{mg} / \mathrm{m}^{2}$, and $150 \mathrm{mg} / \mathrm{m}^{2}$. Evaluation of the safety profile of PIPAC and the associated clinico-pathologic responses at each dose would allow us to identify an optimal dose that balances efficacy with safety and toxicity.

\section{Postoperative follow up}

Each PIPAC is performed in an inpatient elective setting with the subject admitted on the same day as the PIPAC procedure. Patients are admitted for a minimum of 3 days after PIPAC procedure for monitoring and evaluations. Subsequent evaluations are done outpatient. Details on the postoperative evaluations performed are listed in the study schedule (Table 2).

\section{Post study follow up and procedures}

Patients who have ended treatment should be continuously followed for tumour response as per the primary clinician. Patients who demonstrate response to PIPAC with oxaliplatin can be considered for continued PIPAC beyond the first two PIPAC procedures off-trial.

\section{Criteria for withdrawal}

Participants have the right to withdraw at any point during treatment without prejudice. The investigator or regulatory authority can discontinue an individual's participation in the trial at any time if medically or otherwise necessary. The rejection to participate in the study or withdrawal will not compromise with the treatment rendered to the patient and the patient will still receive standard of care treatment. If voluntary withdrawal occurs, the subject will be asked, if willing, to continue scheduled evaluations, complete an end of study evaluation, and be given appropriate care under medical supervision until the symptoms of any adverse event resolve or the subject's condition becomes stable.

\section{Adverse events and safety monitoring plan}

An adverse event (AE) is considered serious (SAE) if it results in death due to any cause, persistent or significant incapacity, or substantial disruption of the ability to conduct normal life functions, hospitalization or prolongation of hospitalization, or an immediately lifethreatening adverse event. The risks of PIPAC can be related to surgical complications as well as to AEs from the intraperitoneal administration of chemotherapy. 
An independent data and safety and monitoring board (DSMB) has been formed to monitor data and safety. The DSMB consists of three subject matter experts who are not part of the study team. The DSMB's purpose is to mitigate the risks from this trial should any unexpected serious adverse effects surface. A report is submitted to the DSMB at the completion of each cohort dose level for review, with approval from the DSMB required prior to the escalation of dose to the next cohort.

\section{Outcome measures}

Baseline details, clinical features, details of surgical treatment, histology, conclusive staging, follow up, and adjuvant therapy details of study subjects will be collected and subsequently summarized.

Surgical complications will be monitored and graded according to the updated Clavien-Dindo Classification [8].

Toxicity will be monitored and graded according to the National Cancer Institute - Common Terminology Criteria for Adverse Events [9]. DLT are defined as grade 3 or higher toxicity which are attributable to the study treatment during the first 28 days of therapy. The maximum-tolerated dose (MTD) is defined as the dose at which fewer than one-third of patients experience a DLT. If multiple toxicities are seen in a study subject, the presence of DLT should be based on the most severe toxicity experienced. DLT definition will use data obtained from first PIPAC cycle only and any toxicities observed subsequently will be reported as AE/SAE.

Clinical response will be assessed according to intraoperative Peritoneal Cancer Index (PCI) [10] during each PIPAC procedure, and with computed tomography using Response Evaluation Criteria In Solid Tumour (RECIST) [11].

Pathological response will be assessed according to the Peritoneal Regression Grade Score (PRGS) [12].

Quality of life will be assessed according to the European Organisation for Research and Treatment of Cancer Quality of Life Questionnaire (EORTC) QLQ-C30 [13].

Patient samples (blood, peritoneal tumour cells, and peritoneal fluid) will be stored in a tissue bank. These samples will be stored with the intent to perform a variety of different molecular analyses to identify genetic biomarkers that are associated with carcinogenesis, cancer progression, response, and resistance to therapy.

\section{Handling and storage of data and documents}

The research data is coded to protect the study subject's confidentiality, and the links between the subject's identifiers and the codes are stored separately from the research data. According to the PI institution's research policy, the essential documents will be retained for a minimum storage of 6 years after the completion of the clinical trial.

\section{Dissemination and publication}

The study is investigator-initiated. Independent of the outcome, the results of the study will be published in international peer-reviewed scientific journals. Patient data will be presented anonymously in any publication or scientific journal.

\section{Discussion}

Peritoneal metastasis is one of the most common and challenging complications of GI cancers, portending a poor and debilitating prognosis with poor response to current systemic treatments [14, 15]. Intraperitoneal (IP) administration of anticancer drugs enables an extremely high concentration of drugs to directly contact the target cancer lesions in the peritoneal cavity. In patients with histologically proven unresectable or recurrent gastric cancer limited to the peritoneum and/or cancer cells in peritoneal cytology, the combination of IP paclitaxel with systemic chemotherapy has been shown to result in a median survival time of 23.6 months [16]. However, its effectiveness is limited by the intraperitoneal distribution and penetration of the drug [17]. Results from a recently completed phase III trial (PHOENIX-GC trial) comparing IP regimen with systemic chemotherapy vs. systemic therapy alone in Japan [18] are eagerly awaited. PIPAC seems to have the added advantage of pressurized aerosolization (better distribution and penetration), while still maintaining the traditional advantages of intraperitoneal chemotherapy (higher intratumoural concentrations as compared to systemic chemotherapy, less systemic toxicity). Each PIPAC treatment is performed laparoscopically, allowing for direct visualization of the peritoneal cavity and repeated peritoneal biopsies to assess for histopathologic response to guide therapy. Furthermore, it does not have the associated complications and technical issues associated with intraperitoneal ports (infection, port migration/blockage). This makes PIPAC a very 
appealing and promising technique. However, there is a dearth of phase I studies in the literature, as basic pharmacokinetic data, toxicity profile, and optimal dose is still lacking. We seek to answer these questions in this phase I trial for PIPAC with oxaliplatin, such that an optimal dose can be found, which balances toxicity with efficacy.

\section{Conclusions}

This phase I study utilizes an evidence-based approach to potentially identify the optimal dose for PIPAC with oxaliplatin to provide a scientific basis for future studies on PIPAC with oxaliplatin, such that the benefits of this novel and promising intraperitoneal chemotherapy delivery technique can be maximized.

Author contributions: All the authors have accepted responsibility for the entire content of this submitted manuscript and approved submission.

Research funding: This work is supported by the National Medical Research Council (NMRC) Centre Grant Programme for the National University Cancer Institute, Singapore (NCIS), National University Health System. (CG12Aug12).

Employment or leadership: None declared.

Honorarium: None declared.

Competing interests: The funding organization(s) played no role in the study design; in the collection, analysis, and interpretation of data; in the writing of the report; or in the decision to submit the report for publication.

\section{References}

1. Solaß W, Hetzel A, Nadiradze G, Sagynaliev E, Reymond MA. Description of a novel approach for intraperitoneal drug delivery and the related device. Surg Endosc Other Interv Tech. 2012;26:1849-55.

2. Solass W, Herbette A, Schwarz T, Hetzel A, Sun JS, Dutreix M, et al. Therapeutic approach of human peritoneal carcinomatosis with Dbait in combination with capnoperitoneum: proof of concept. Surg Endosc. 2012;26:847-52.

3. Nadiradze G, Giger-Pabst U, Zieren J, Strumberg D, Solass W, Reymond MA. Pressurized intraperitoneal aerosol chemotherapy (PIPAC) with low-dose cisplatin and doxorubicin in gastric peritoneal metastasis. J Gastrointest Surg. 2016;20 (2):367-3.

4. Robella M, Vaira M, De Simone M. Safety and feasibility of pressurized intraperitoneal aerosol chemotherapy (PIPAC) associated with systemic chemotherapy: an innovative approach to treat peritoneal carcinomatosis. World J Surg Oncol. 2016;14:128.
5. Solass W, Kerb R, Mürdter T, Giger-Pabst U, Strumberg D, Tempfer C, Zieren J, et al. Intraperitoneal chemotherapy of peritoneal carcinomatosis using pressurized aerosol as an alternative to liquid solution: first evidence for efficacy. Ann Surg Oncol. 2014;21:553-59.

6. Demtröder C, Solass W, Zieren J, Strumberg D, Giger-Pabst U, Reymond MA. Pressurized intraperitoneal aerosol chemotherapy with oxaliplatin in colorectal peritoneal metastasis. Color Dis. 2016;18:364-71.

7. Hübner M, Teixeira Farinha H, Grass F, Wolfer A, Mathevet $P$, Hahnloser D, et al. Feasibility and safety of pressurized intraperitoneal aerosol chemotherapy for peritoneal carcinomatosis: a retrospective cohort study. Gastroenterol Res Pract. 2017;2017:6852749.

8. Dindo D, Demartines N, Clavien PA. Classification of surgical complications: a new proposal with evaluation in a cohort of 6336 patients and results of a survey. Ann Surg. 2004;240:205-13.

9. National cancer institute - common terminology criteria for adverse events (CTCAE). 2010;2009:0-71. Available from: http://ctep.cancer.gov/protocolDevelopment/electronic_appli cations/docs/ctcaev3.pdf

10. Pestieau SR, Belliveau JI, Griffin H, Stuart OA, Sugarbaker PH. Pharmacokinetics of intraperitoneal oxaliplatin: experimental studies. J Surg Oncol. 2001;76:106-14.

11. Eisenhauer EA, Therasse P, Bogaerts J, Schwartz LH, Sargent D, Ford $R$, et al. New response evaluation criteria in solid tumours: revised RECIST guideline (version 1.1). Eur J Cancer. 2009;45:228-47.

12. Solass W, Sempoux C, Carr NJ. Peritoneal sampling and histological assessment of therapeutic response in peritoneal metastasis: proposal of the peritoneal regression grading score (PRGS). Pleura and Peritoneum. 2016;1:99-107.

13. Aaronson NK, Ahmedzai S, Bergman B, Bullinger M, Cull A, Duez $\mathrm{N}$, et al. The European organization for research and treatment of cancer QLQ-C30: a quality-of-life instrument for use in international clinical trials in oncology. J Natl Cancer Inst. 1993;85:365-76.

14. Yoo CH, Noh SH, Shin DW, Choi SH, Min JS. Recurrence following curative resection for gastric carcinoma. $\mathrm{Br}$ J Surg. 2000;87:236-42.

15. Franko J, Shi Q, Meyers JP, Maughan TS, Adams RA, Seymour MT, et al. Prognosis of patients with peritoneal metastatic colorectal cancer given systemic therapy: an analysis of individual patient data from prospective randomised trials from the analysis and research in cancers of the digestive system (ARCAD) database. Lancet Oncol. 2016;17:1709-19.

16. Ishigami H, Kitayama J, Kaisaki S, Hidemura A, Kato M, Otani K, et al. Phase II study of weekly intravenous and intraperitoneal paclitaxel combined with S-1 for advanced gastric cancer with peritoneal metastasis. Ann Oncol. 2010;21:67-70.

17. Prada-Villaverde A, Esquivel J, Lowy AM, Markman M, Chua T, Pelz J, et al. The American society of peritoneal surface malignancies evaluation of HIPEC with mitomycin C versus oxaliplatin in 539 patients with colon cancer undergoing a complete cytoreductive surgery. J Surg Oncol. 2014;110:779-85.

18. Kono K, Yong WP, Okayama H, Shabbir A, Momma T, Ohki S, et al. Intraperitoneal chemotherapy for gastric cancer with peritoneal disease: experience from Singapore and Japan. Gastric Cancer. 2017;20:122-27. 